\title{
Rapprocher l'élu et le citoyen. La " proximité » dans le débat sur la limitation du cumul des mandats (1998-2000)
}

\section{Rémi Lefebvre}

\section{(2) OpenEdition Journals}

Édition électronique

URL : https://journals.openedition.org/mots/127

DOI : $10.4000 /$ mots. 127

ISSN : 1960-6001

Éditeur

ENS Éditions

\section{Édition imprimée}

Date de publication : 1 mars 2005

Pagination : 41-57

ISBN : 2-84788-077-1

ISSN : 0243-6450

\section{Référence électronique}

Rémi Lefebvre, «Rapprocher l'élu et le citoyen. La « proximité » dans le débat sur la limitation du cumul des mandats (1998-2000) ", Mots. Les langages du politique [En ligne], 77 | 2005, mis en ligne le 31 janvier 2008, consulté le 23 avril 2022. URL : http://journals.openedition.org/mots/127 ; DOI : https://doi.org/10.4000/mots.127 


\section{Rapprocher l'élu et le citoyen. La «proximité » dans le débat sur la limitation du cumul des mandats (1998-2000)}

La proximité est devenue dans le champ politique une référence obligée, un mot d'ordre unanimement mis en avant par le personnel politique et pensé comme un remède à la crise du politique. Dans le contexte de plus en plus intériorisé par les élus d'une crise de la représentation, la proximité est perçue comme une manière de ressourcer la légitimité politique et de refonder le lien représentatif en ancrant les pratiques politiques dans l'immédiateté et la concrétude des problèmes rencontrés par les citoyens ${ }^{1}$. Elle est devenue ainsi un parangon de modernité. La modernisation politique a longtemps renvoyé à un processus de dépersonnalisation des relations politiques et d'éradication des relations clientélaires de dépendance personnelle. Se parer de la modernité politique, ce fut longtemps, comme encore aux temps fondateurs de la Cinquième République, s'attaquer aux féodalités locales et aux relations particularistes qui faisaient écran à l'intérêt général ${ }^{2}$. Moderniser la vie politique, c'est au contraire, aujourd'hui, multiplier les relations individualisées et les contacts entre représentants et représentés. D’aucuns ont pu voir, dans le thème de la proximité et la valorisation des relations personnalisées, une nouvelle ruse de l'Histoire : "Ce que l'on croyait pouvoir définir comme un archaïsme des sociétés méditerranéennes devient un facteur de modernité. ${ }^{3}$ On comprend dans ces conditions que la politique locale, espace d'une proximité naturalisée, soit investie de la mission de réhabiliter la politique et que de nouveaux flux de légitimité circulent entre local et national. La fortune de ce

1. Sur ce point, R. Lefebvre, 2001, "Rhétorique de la proximité et "crise de la représentation" ", Cahiers lillois d'économie et de sociologie, $\mathrm{n}^{\circ} 35$, Paris, L'Harmattan, p. 111-132 et R. Lefebvre, 2004, "Quand légitimité rime avec proximité», Mouvements, n³2, p.56-65. Pour B. Lacroix, si les hommes politiques se sont saisis du thème de la crise de la représentation et l'ont même promu, contribuant à le diffuser, c'est pour mieux réaffirmer la fonctionnalité et la nécessité de la représentation («Existe-t-il une crise de la démocratie représentative en France aujourd'hui ? Éléments pour une discussion sociologique du problème», dans D. Rousseau (dir.), 1994, La démocratie continue, Paris, LDJD, p. 40-59).

2. D. Dulong, 1997, Moderniser la politique. Aux origines de la Ve République, Paris, L'Harmattan.

3. P. Alliès, 2002, "L'arrivée d'une droite de courtoisie », Le Monde, 21 mars.

Université Lille 2, CERAPS-CNRS, rlefebvre@hp-sc.univ-lille2.fr 
discours de la proximité est à la mesure de son indétermination, de son flou définitoire et de sa malléabilité. Le caractère équivoque de cette notion apparait comme une condition essentielle de ce succès social. Le mot, fortement polysémique, fonctionne sur le mode de l'implicite et ses ambigüités prêtent à des usages très différenciés. La proximité peut être d'ordre physique, géographique, temporel ou morphologique, mais aussi d'ordre social ou affinitaire (le proche est le familier, ce qui n'est pas étranger). Par ailleurs, les contours du terrain dont se prévalent les élus apparaissent flous. La teneur des réalités locales, substantialisées, dont les élus seraient porteurs est rarement explicitée, de sorte qu'on ne sait pas au juste de quelle étoffe est fait ce terrain avec lequel les élus sont censés faire corps. Une certaine évidence sociale s'attache à l'idée de proximité et à son invocation par les élus.

Cette rhétorique de la proximité a été omniprésente lors du long débat sur la limitation du cumul des mandats, plusieurs fois inscrit à l'ordre du jour parlementaire entre 1998 et 2000. "Nos concitoyens veulent que les représentants se consacrent entièrement à leur mandat. Ils ont besoin de retrouver confiance dans la vie politique et en particulier en ceux qui l'animent. Limiter strictement le cumul des mandats est ainsi devenu une priorité » : c'est en ces termes que, le 19 juin 1997, dans sa déclaration de politique générale à l'Assemblée nationale, Lionel Jospin présente la limitation du cumul des mandats, qu'il appelle de ses vœux. Cette mesure participe alors d'une démarche de rénovation des institutions et des pratiques politiques qui prend le label de «modernisation de la vie politique» et dont le gouvernement cherche à faire une marque distinctive. Ce dessein politique auquel les médias donnent une large publicité reçoit la légitimation d'un certain nombre de politistes et de constitutionnalistes, opposés au cumul, qui contribuent à la remise à l'agenda de cet enjeu en 1997-1998. Yves Mény présente ainsi cette réforme comme «la mère de toutes les réformes » en matière de rénovation des institutions, Lionel Jospin évoque quant à lui «le premier fil de la pelote» ${ }^{4} .$. Les projets de loi, adoptés par le conseil des ministres le 8 avril 1998, poursuivent quatre objectifs : interdire à un parlementaire français, député ou sénateur, d'être en même temps membre du Parlement européen, interdire à tout élu de présider deux exécutifs locaux, interdire à un élu de détenir plus de deux mandats et surtout, cœur de la réforme, couper le lien entre la fonction législative et la fonction exécutive locale en interdisant à tout député ou sénateur d'être en même temps maire, président de conseil général ou président de conseil régional. Le projet gouvernemental fait l'objet de quatre lectures parlementaires entre avril 1998 et mars 2000. Le Sénat bloque, à trois reprises, le débat

4. Voir B. Roman, 2000, La fin du cumul des mandats, Paris, Bruno Leprince, p. 114. 
sur le projet de loi organique mais ne peut s'opposer à l'adoption définitive de deux textes de loi le 8 mars 2000.

C'est à l'analyse de ce débat que l'on voudrait ici s'attacher. Au cours de celui-ci, partisans et opposants à la réforme ont fortement mis en avant dans leurs argumentaires respectifs la proximité comme une nécessité indiscutable, un horizon indépassable. Un des enjeux des échanges a été la captation des profits symboliques attachés à une exigence conçue comme incontournable et pesant sur le personnel politique alors qu'il cherche à (auto) règlementer ses pratiques. Il ne s'agit pas, dans le cadre de cette étude, de mettre à jour l'économie générale du débat et l'ensemble des discours de justification qu'il a mis en jeu, mais seulement d'envisager la manière dont la thématique de la proximité, fortement plastique, a été saisie et mise en forme par les stratégies argumentatives en présence. Dès lors que la proximité est enjeu de luttes symboliques, quelle définition en est promue ? Quel contenu est donné à la relation dite de proximité ? On s'attachera principalement à l'analyse des débats parlementaires (Assemblée nationale et Sénat). Rares sont les débats portant directement, dans les arènes politiques, sur les pratiques des professionnels de la politique. Peu fréquentes sont les occasions où les hommes politiques sont invités ou contraints à évoquer leur métier, la spécificité de leurs tâches et de leur activité. Une des caractéristiques les plus prégnantes du métier politique est qu'il demeure largement indéterminé, faiblement codifié. Le débat sur le cumul, qui contraint les représentants à débattre et à légiférer sur leurs propres pratiques et donc à les justifier publiquement, à en rendre compte, constitue à ce titre un contexte tout à fait intéressant où les joueurs sont contraints de mettre à plat les règles du jeu ou, du moins, de les expliciter. L'analyse de ce type de débats apparait d'autant plus heuristique qu'ils peuvent remplir une fonction d'explicitation de règles du jeu le plus souvent implicites 5 .

La question de la légitimité du jeu politique représentatif et la manière d'en restaurer le crédit ont été au centre des confrontations. La thématique de la crise du politique ou de la représentation ont surdéterminé des débats qui apparaissent dominés par un acteur collectif sans cesse mobilisé et parlé : l'opinion publique. Les professionnels de la politique sont statutairement contraints de ne représenter que les intérêts des citoyens et donc de dénier leurs propres intérêts, y compris (et peut-être surtout) lorsqu'ils sont très directement mis en jeu ${ }^{6}$. Diverses conceptions de la proximité ou du terrain

5. Sur cette question, voir P. Lehingue, 1999, «Vocation, art, métier ou profession ? Codification et étiquetage des activités politiques», dans M. Offerlé (dir.), La profession politique, Paris, Belin, p. $93-134$.

6. À propos des débats parlementaires, M. Abélès écrit : «On y discute entre soi tout en s'adressant à des tiers» (M. Abélès, 2000, Un ethnologue à l'Assemblée, Paris, Odile Jacob, p. 252). 
ont été mobilisées de manière concurrente en étant adossées à des constructions contradictoires de la demande sociale émanant des citoyens. La majorité parlementaire présente la réforme comme une priorité exprimée par l'opinion, découlant d'une exigence de disponibilité. L'opposition dénonce le risque d'une désarticulation entre le local et le national et donc d'une césure avec la France réelle et le terrain, ce que les Français ne souhaitent pas. Tout se passe pourtant comme si, par-delà les définitions plurielles de la proximité que le débat donne à voir et met en jeu, se dégageait une conception partagée des règles du jeu politique. Au-delà des désaccords affichés et dramatisés, c'est un accord tacite et collusif sur une manière de faire de la politique et d'en restaurer la légitimité qui semble se manifester.

\section{Les partisans de la réforme : une réponse à la « crise du politique»}

Les promoteurs de la loi (rapporteur, ministres, parlementaires) s'appuient sur un argumentaire qui fait appel à plusieurs registres de justification. Le cumul des mandats est présenté comme un facteur explicatif d'un certain nombre de dysfonctionnements de l'ingénierie institutionnelle. Il organiserait la confusion d'intérêts et nuirait à la transparence des responsabilités, affaiblirait le Parlement en contribuant à l'absentéisme parlementaire, renforcerait la technocratie. La limitation du cumul doit par ailleurs contribuer à un renouvellement du personnel politique et permettre une meilleure respiration de la démocratie. Mais la problématique institutionnelle tend à être recouverte par une thématique plus large, particulièrement prégnante dans les discours de la majorité, celle de la crise de la représentation. La mesure est présentée surtout comme un moyen de réhabiliter la politique. L'invocation de la crise du politique fonctionne comme un argument d'autorité que les défenseurs de la réforme ne cesseront tout au long du débat de brandir et d'instrumentaliser, mettant le débat sous surveillance de l'opinion?.

\section{Une stratégie d'appel à l'opinion}

Tout au long du débat, les orateurs de la majorité s'adossent à l'opinion et invoquent la crainte que les élus n'accroissent leur propre discrédit par leur éventuel refus de remettre en cause la pratique du cumul. L'argumentaire des partisans du projet peut se résumer en ces termes : l'opinion publique est favo-

7. Pour reprendre l'expression de A. Collovald, B. Gaïti, 1990, «Discours sous surveillances. Le social à l'Assemblée», dans D. Gaxie (dir.), Le social transfiguré, Paris, PUF-CURAPP, p. 5-50. 
rable très majoritairement à la réforme qui doit rapprocher les élus des citoyens; c'est devant cette opinion que les élus de l'opposition doivent se justifier et prendre leurs responsabilités ${ }^{8}$.

Jean-Jacques Queyranne, ministre de l'Intérieur par intérim, présente en ces termes le projet devant le Sénat (séance du 27 octobre 1998) :

Il faut savoir bousculer des habitudes et revenir sur des situations acquises. En apportant ainsi la preuve de sa capacité à réformer la représentation politique, le Parlement, j'en suis sûr, contribuera à rétablir la confiance des citoyens dans leurs institutions.

Les propos de Guy Allouche, sénateur socialiste, sont emblématiques de la posture adoptée par la majorité (séance du 27 octobre 1998) :

Les élus de la nation, qui devraient être des exemples, sont jour après jour mis en cause par un discours antipolitique et antiparlementaire qu'alimente la déprime économique et sociale [...]. Il est devenu urgent d'envoyer un signe fort de notre volonté de clarifier nos structures politiques. La démocratisation et la modernisation de notre vie politique sont désormais des enjeux majeurs. L'interdiction de cette grande spécificité hexagonale qu'est le cumul des mandats et des fonctions exécutives fait partie des décisions susceptibles de désamorcer la crise du politique [...]. La limitation du cumul des mandats est l'une des réformes majeures à entreprendre pour réconcilier les Français avec leurs élus. Elle est l'une des plus symboliques pour la simple raison qu'elle est directement compréhensible par l'opinion [...]. En 1995, Lionel Jospin en avait fait un des axes de sa campagne présidentielle, et il s'y est de nouveau engagé en 1997. Le résultat des urnes laisse penser que les Français sont attachés à cet objectif. Ce mouvement d'opinion est porté par une puissante aspiration à la transparence de la vie politique, à l'égalité et à l'ouverture du monde politique à la société civile.

À mesure que le débat avance et que la perspective d'un refus du Sénat se dessine, la stratégie de la dramatisation et de la scandalisation ${ }^{9}$ devient plus appuyée. L'opinion, constamment invoquée, mobilisée, parlée, est posée en recours ultime. Devant des sénateurs inflexibles, Guy Allouche déclare (séance du 2 mars 2000) :

Chaque fois qu'il s'agit de moderniser notre vie publique et institutionnelle, de traduire dans la loi les avancées sociales et culturelles, le Sénat se montre frileux, très en retrait, voire franchement hostile. Le parti pris du Sénat est celui du laisser-faire, peut-être même celui du parti de la loi naturelle. Ma conviction profonde est que l'opinion publique condamnera, une fois encore, le refus de la droite sénatoriale d'accompagner ces évolutions, tout comme elle a déjà sévèrement jugé son rejet des autres projets de réforme de société [...]. Le gouvernement a décidé de prendre acte de la position de refus du Sénat. Mes chers collègues, prendre acte ne signifie

8. Sur la question du rapport de l'opinion à cette question, voir L. Olivier, 1998, «La perception du cumul des mandats. Restrictions contextuelles et politiques à un apparent consensus », Revue française de science politique, $\mathrm{n}^{\circ}$ 48-6, décembre, p. 50-70.

9. Sur cette notion, voir M. Offerlé, 1994, Sociologie des groupes d'intérêt, Paris, Montchrestien. 
pas renoncer, c'est une simple pause dans la mise en œuvre d'une réforme voulue et attendue par les Français. Ce n'est que partie remise. Face à cette opposition résolue, j'allais dire frontale, nous emprunterons, le moment venu, une voie encore plus démocratique de contournement de l'adversaire.

\section{Les figures du rapprochement : identité et disponibilité}

C'est dans ce cadre que se loge le discours de la proximité. Si l'opinion est favorable à la réforme, c'est qu'elle souhaite des élus plus «proches », c'est-àdire disponibles. Jean-Jacques Queyranne se fait le porte-parole de cette exigence : «Nos concitoyens souhaitent que leurs élus se consacrent pleinement à leurs mandats » (séance du 27 octobre 1998). "La vitalité de notre démocratie, le renforcement du pacte républicain passent par le rapprochement indispensable du peuple et de ses représentants» (Guy Allouche, séance du 19 octobre 1999). La limitation du cumul doit renforcer la proximité des élus, elle va donc dans le bon sens. Pour le sénateur communiste, Robert Bret (séance du 27 octobre 1998) : "Limiter le cumul aboutira à resserrer les liens entre l'élu et le citoyen en permettant au premier de se consacrer pleinement à sa fonction élective.» L'élu pourra donner à cette proximité une dimension nouvelle. La réforme obéit à ce que le député socialiste, Bernard Roman, rapporteur de la loi, énonce comme «le principe de disponibilité ${ }^{10}$. Guy Allouche (séance du 28 octobre 1998) déclare :

On ne me convaincra pas que le titulaire d'un mandat exécutif local qui passe son temps à traiter des dossiers avec la technostructure locale - et dans mon esprit, ce terme n'est pas péjoratif - a le temps d'aller constamment au-devant de ses électeurs. Ayons le courage de le dire : il est évident qu'il ne le fait pas ! [...]. En fait, la proximité sera d'autant plus étroite que le parlementaire n'aura pas en charge la gestion d'une collectivité territoriale.

La véritable proximité, en somme, c'est l'identité sociale entre les citoyens et leurs représentants. C'est un excès d'altérité entre représentants et représentés qu'il s'agit de conjurer. Une première figure du rapprochement se dessine si l'on suit les usages faits par la majorité du mot «proximité».

Pour Bernard Roman (Assemblée nationale, séance du 26 mai 1998), il faut «instaurer une relation plus claire, plus transparente, plus directe et plus exclusive entre le citoyen et l'élu».

Si un député ou un sénateur revenant dans sa circonscription après une semaine de session à l'Assemblée nationale consacre deux ou trois jours à être dans sa permanence, à être sur le terrain, à vivre tout simplement comme les électeurs de sa

10. Rapport parlementaire de B. Roman, Assemblée nationale, mai 1998. On y lit : «La loi doit permettre aux députés et aux sénateurs d'être davantage présents sur le terrain, au contact direct avec leurs électeurs» (p. 33). 
circonscription, à aller chercher ses enfants ou ses petits-enfants à l'école, à vivre normalement comme des citoyens vivent, eh bien je crois que là, réellement, le lien avec la réalité locale sera non seulement maintenu mais, bien plus, renforcé ${ }^{11}$.

Le rapporteur déclare dans le même sens dans un journal lillois :

La révolution culturelle que nous engageons, c'est de faire en sorte que la société politique ressemble à la société elle-même. Les élus doivent ressembler à leurs électeurs. Parler comme eux, s'habiller comme eux, rouler dans les mêmes voitures ${ }^{12}$.

Pour le député Arnaud Montebourg (séance du 27 mai 1998), «les élus seront plus proches des électeurs car ils leur ressembleront plus». La limitation du cumul, on le voit, est justifiée pour partie au nom de la proximité et d'une conception de la représentation comme «miroir $»^{13}$. La nécessité de préserver le rapport au terrain est très rapidement avalisée par les parlementaires de la majorité (on y reviendra). C'est que l'opposition a fait de la question du terrain une des problématiques centrales du débat dont elle a contribué à définir les enjeux. À la volonté de l'opinion est opposée la nécessité du terrain, ellemême dictée par l'opinion.

\section{Les opposants au projet : rester en prise avec le «terrain " $^{14}$}

La stratégie argumentative des opposants au projet (essentiellement la droite parlementaire) relève elle aussi de multiples dimensions qu'il n'est pas ici dans notre propos d'épuiser. Le cumul est présenté comme un aspect quasi intangible de la culture et de la tradition politiques françaises qui s'enracine dans la tradition de la centralisation étatique. Pour Jacques Brunhes, «la ténacité de cette tradition est un indice de sa fonctionnalité dans le système politique » (séance du 26 mai 1998, Assemblée nationale), le cumul jouant un rôle compensateur face au pouvoir de l'État ${ }^{15}$... De multiples orateurs font valoir également la «faiblesse des partis politiques» pour expliquer et justifier le phénomène. Mais l'argumentaire des partisans du cumul des mandats s'organise surtout autour de la nécessité sans cesse martelée de préserver le lien des élus avec le terrain et les citoyens.

\footnotetext{
11. L'Autrement dit, 24 octobre 1997.

12. Nord-Éclair, 26 et 27 avril 1998.

13. Voir P. Rosanvallon, Le peuple introuvable, Paris, Gallimard, 1998.

14. Les mots proximité et terrain sont employés de manière quasi interchangeable à gauche et à droite, les élus de l'opposition utilisant le mot terrain plus régulièrement.

15. Voir J. Rovan, 1998, Le Monde, 30 avril. Sur l'invention et la genèse historique de ce registre «indigène» de justification qui a pu s’appuyer sur le savoir sociologique, voir G. Marrel, 2003, L'élu et son double. Cumul des mandats et construction de l'État républicain en France du milieu $d u x i x^{e}$ siècle au milieu du xxe siècle, thèse de science politique, Université Grenoble 2.
} 


\section{Ne pas couper le lien local-national}

Le projet prévoit, rappelons-le, d'empêcher le cumul d'un mandat de parlementaire avec celui de direction d'un exécutif local. Ce serait, selon les opposants au projet, priver et déconnecter le Parlement des réalités du terrain et du sens aiguisé des "réalités locales» dont les élus seraient les traducteurs à Paris. Le projet contrevient à l'exigence de proximité alors que «l'intérêt porté à la gestion de proximité n'a sans doute jamais été aussi prononcé que dans la société actuelle» (Bernard Plasait, sénateur RPR, séance du 27 octobre 1998).

C'est le «risque de fracture entre le pays réel et le pays légal» qu'encourt le système politique (Josselin de Rohan, sénateur, séance du 19 octobre 1999). Lors de son discours au congrès de l'Association des maires de France, le 20 novembre 1997, Jacques Chirac avait, en amont du débat, exprimé son attachement à l'expérience irremplaçable des élus locaux : «Il est essentiel que celles et ceux qui ont la lourde responsabilité d'élaborer la loi ne soient pas coupés des réalités du terrain. Telle est notre culture. Il est essentiel, et j'en fais moi-même l'expérience en tant qu'élu de la Corrèze, que nos députés et nos sénateurs puissent rester à l'écoute de la France.» «Il n'est pas imaginable qu'un parlementaire puisse être en lévitation par rapport au terrain» (Bernard Joly, Sénat, séance du 27 octobre 1998). Les mandats locaux du député, irremplaçables, «lui permettent, sans sondages, de prendre le pouls de la population et de comprendre les angoisses, les inquiétudes, les revendications de la société civile comme de toute la population. $\gg^{16}$

Qui peut sincèrement prétendre que le chef d'un exécutif local serait en mesure d'appréhender plus concrètement les intentions ou les effets d'une loi sur son territoire s'il lui était dorénavant interdit de participer à son élaboration ? [...] Un parlementaire limité à ses fonctions législatives ne serait plus, en réalité, qu'un technicien de l'élaboration des lois, puisque cette disposition, en toute logique, le couperait de toute vie professionnelle. (Georges Tron, séance du 26 mai 1998, Assemblée nationale)

La politique, loin de n'être qu'un exercice cérébral, est une expérience presque instinctuelle, une activité qui engage un rapport charnel avec le territoire et les citoyens (voir l'intervention de Pierre Albertini, séance du 26 mai 1998, Assemblée nationale). Il faut donc préserver le lien physique du parlementaire avec son territoire, gage d'un rapport concret à la politique. Ces réalités du terrain ne peuvent être appréciées que par la maitrise d'un exécutif local. Les rapports que le député entretient avec sa circonscription ne sauraient suffire. André Santini s'interroge :

16. T. Cornillet, 1997, "Que tout parlementaire puisse cumuler avec un seul mandat exécutif», Revue politique et parlementaire, $\mathrm{n}^{\circ} 991$, novembre-décembre. 
Comment peut-on imaginer sérieusement qu'au cours d'une permanence, de banquets ou de quelques cérémonies frivoles, un parlementaire soit réellement capable de comprendre la véritable dimension des problèmes d'une circonscription, des difficultés quotidiennes des électeurs et des besoins de ses concitoyens ? Privé des responsabilités locales, que représente-t-il, qui représente-t-il ? [...] La campagne des dernières législatives s'est faite sur le thème de la proximité. On va rompre aujourd'hui ce lien qui permet aux élus locaux d'écouter leurs administrés pour les faire entendre au plan national ${ }^{17}$.

Le mandat local fait partie d'un cursus traditionnel et républicain qu'il faut préserver parce qu'il garantit un apprentissage sur le tas dont tire profit le travail législatif. «En fait, argumente Michel Crépeau ${ }^{18}$, se pose un problème de pouvoir et il n'est pas de pouvoir sans légitimité. Or l'exception française, dont nous devons être fiers, réside dans le fait qu'il existe une double légitimité dans ce pays depuis le fond des âges, depuis la nuit des temps, car cela tient à notre histoire : une légitimité qui vient du savoir, des grandes écoles, c'està-dire une légitimité qui vient du haut; une légitimité qui vient du bas, de l'élection, des pouvoirs locaux. Je crois qu'il est indispensable que ces deux légitimités se rencontrent au niveau du Parlement» (Assemblée nationale, $2^{\mathrm{e}}$ séance du 26 mai 1998).

Le cumul permet, par le biais des élus, d'articuler intuitu personae l'échelon local et l'échelon national; il assure une dimension duale (local/national) et incarnative de la représentation qu'il faut maintenir. L'argument de l'ancrage local tend à être opposé à tous les arguments de la majorité comme s'il était d'autorité. Aux élus de gauche qui pointent les risques de localisme et de sensibilité trop forte aux intérêts particuliers, Jean-Paul Delevoye, alors président de l'Association des maires de France, répond (séance du 27 octobre 1998, Sénat) : «Je crois que, contrairement à ce que vous dites, c'est en renforçant l'assise locale d'un élu qu'on lui permet de conforter son indépendance et son objectivité.» Pourtant, le contenu même de cette relation de proximité, établi sur le plan local, n'est guère explicité à la faveur du débat. Tout se passe comme si elle allait de soi, comme si son intensité ne pouvait être contestée, comme si son caractère prétendument démocratique n'appelait pas questionnement. Les élus locaux, et les maires tout particulièrement, s'autodésignent comme les interprètes les plus directs, les plus fidèles et les plus constants de la base politique. Les maires font naturellement corps avec les citoyens. La droite, on le voit, puise dans les ressources symboliques de l'idéologie professionnelle des maires, élus qui font de leur proximité un attribut central et incontesté de légitimité. Plusieurs parlementaires de gauche

17. A. Santini, 1997, «Deux mandats, sinon rien!», Revue politique et parlementaire, $\mathrm{n}^{\circ}$ 991, novembre-décembre.

18. Député radical, il ne soutient pas le projet gouvernemental. 
font valoir que le rapport au terrain du député est assuré de fait par le mode de scrutin par circonscription qui implique l'ancrage local ${ }^{19}$. Mais cet argument se révèle d'une faible portée tant la proximité du maire parait irremplaçable.

Les élus de l'opposition tentent d'imposer la croyance qu'à travers les députés-maires, leurs actes et leurs paroles, hors de tout travail politique de traduction, c'est le pays qui s'exprime fidèlement ${ }^{20}$. Il y aurait un degré zéro de la délégation de parole et les élus locaux seraient les premiers maillons d'un véritable continuum de représentation. À travers le travail des élus s'opère un processus de consultation démocratique permanent dont le cumul des mandats permet de porter les effets et les résultats au niveau national. Ce processus ascendant ne saurait donc être remis en cause, toujours selon les opposants à la réforme. Sont occultés ainsi les effets de dépossession inhérents au travail de représentation. Les élus locaux s'autorisent d'une base politique qui n'existe pas indépendamment du travail politique de représentation qui la constitue comme tel. À travers le débat est ainsi à l'œuvre le mythe de la base au cœur des mécanismes et de l'efficace de la représentation politique ${ }^{21}$.

\section{Le recours à l'opinion : la construction d'une demande sociale de proximité}

C'est devant le tribunal de l'opinion dont la majorité se fait le porte-parole que les opposants au projet doivent justifier leur position. Ils le font de diverses façons qui traduisent bien, de manière plus générale, l'ambigüité structurelle du rapport des élus à l'opinion. L'opposition conteste d'abord la pression excessive de l'opinion et des médias qui s'exerce sur les parlementaires et met en cause le caractère souverain de leur décision. La réforme soumise aux parlementaires est présentée comme une loi de circonstance dictée par la démagogie encouragée par la logique d'opinion.

Les sénateurs déplorent à de nombreuses reprises les pressions instrumentalisées de l'opinion et «l'ambiance médiatique qui entoure ce débat» (Paul Girod, séance du 27 octobre 1998, Sénat). Bernard Plasait (sénateur RPR, séance du 27 octobre 1998) déclare : «Le gouvernement [...] cède à la démagogie [...]. Je crois que le gouvernement aurait été mieux inspiré en expliquant aux Français que le cumul, c'est avant tout l'addition de tâches souvent

19. «Un parlementaire qui reçoit dans ses permanences ou qui sillonne sa circonscription a toutes les occasions d'être confronté aux problèmes et aux attentes de son électorat sans qu'il ait besoin pour autant de gérer une commune, un département ou une région» (Guy Allouche, séance du 27 octobre 1998, Sénat).

20. Ce type de discours est régulièrement mobilisé par les élus de gauche hors de cette période de débat.

21. C. Suaud, 1984, "Le mythe de la base ", Actes de la recherche en sciences sociales, $n^{\circ}$ 54, p. 5676 et P. Bourdieu, 2001, "Le mystère du ministère », Actes de la recherche en sciences sociales, $\mathrm{n}^{\circ} 140$, p. $7-11$. 
ingrates et souvent indispensables au bon service du citoyen.» Jean-Paul Delevoye (séance du 27 octobre 1998, Sénat) instruit le procès de la démocratie d'opinion :

Notre démocratie ne souffre pas aujourd'hui d'un quelconque cumul des fonctions mais d'une sorte de zapping émotionnel qui perturbe profondément notre électorat, le rendant de plus en plus sceptique à l'égard de la chose publique, voire de l'État, tant il est convaincu que le vice est davantage récompensé que la vertu, tandis que la loi de la rue remplace la loi républicaine et que le débat sur les médias vaut débat dans l'hémicycle.

Les exigences des citoyens sont présentées comme contradictoires :

Les Français, et il suffit d'être député depuis quelques années pour le savoir, veulent deux choses un peu contradictoires. Tout d'abord, avoir un député à leur disposition personnelle, à sa permanence, pour les mariages, les enterrements... Et puis, il y a les fêtes, les cérémonies, toutes les manifestations du week-end. Je n'exagère pas, mes chers collègues, vous le savez, puisque comme moi vous êtes députés depuis un certain temps, voire un temps certain en ce qui me concerne [...]. Les Français veulent donc un député qui soit à la botte de l'électeur et il est vrai que nous sommes à leur service; mais ils veulent aussi le contraire, c'est-à-dire que nous soyons jour et nuit présents à l’Assemblée! (Pascal Clément, troisième séance du 26 mai 1998, Assemblée nationale)

Mais cette position de contestation de l'opinion est difficile à défendre. Les élus contestent alors le monopole que les sondages exercent sur l'expression de l'opinion et s'autoproclament comme des médiateurs plus fidèles. Michèle Alliot-Marie (séance du 27 mai 1998) oppose à la démocratie sondagière une démocratie plus authentique, celle des élus locaux. Ce que révèle l'opinion, abusée par les promoteurs de la lojín $^{22}$, c'est un attachement au terrain et à la proximité. La défense du cumul se fait donc au nom des attentes des électeurs que les élus connaissent bien. Jacques Peyrat, sénateur RPR (séance du 27 octobre 1998), s'en fait l'interprète : «Ce que veulent les Français aujourd'hui, monsieur le ministre, ce sont des élus aussi proches que possible d'eux et de leurs préoccupations afin qu'ils répercutent dans les hautes assemblées leurs attentes, leur volonté et leur espérance.»

Les parlementaires cherchent ensuite à éclairer l'opinion sur les dérives que recèle une limitation plus forte du cumul. Les repoussoirs de la technocratie et de la bureaucratie partisane, figures inversées de la proximité, sont alors brandis. La réforme risque de livrer la démocratie aux partis, à la technocratie ou aux lobbies. Seul le contact direct avec les citoyens permet de se soustraire à l'emprise des appareils partisans. Avec la loi, la dérive vers le scrutin proportionnel et le règne des partis seraient inéluctables :

22. Certains orateurs avancent l'idée que les Français confondent cumul des indemnités et cumul des fonctions. 
Votre raisonnement ne vaut que si l'on coupe le lien électoral du député avec sa circonscription, c'est-à-dire si vous changez le mode de scrutin, et vous n'osez pas dire que votre projet induit l'instauration d'un scrutin proportionnel pour l'élection des députés, c'est-à-dire, in fine, des députés qui seraient désignés par les partis politiques et la fin de tout enracinement local. (Michel Herbillon, troisième séance du 26 mai 1998, Assemblée nationale ${ }^{23}$ )

L'expérience du terrain permet de faire pièce ou échec au pouvoir des fonctionnaires, de fonder face à eux l'autorité des élus :

Que l'on soit membre du gouvernement ou député, notre force réside dans la possibilité de faire appel à notre expérience de terrain pour dire au haut fonctionnaire concerné : dans ma circonscription, dans mon département, dans ma commune, cela ne se passe pas ainsi que vous le dites, mais comme ça. (Pascal Clément, Assemblée nationale, séance du 26 mai 1998)

Le cumul enfin permet de mettre à distance les lobbies.

Le député sans autre mandat exécutif aura plus difficilement cette exigence du concret et le souci de la mise en œuvre de ses décisions. Il sera moins attentif aux enjeux et conflits d'intérêts qui existent dans notre société. Dès lors, les risques de manipulation par les lobbies politiques, professionnels ou catégoriels s'en trouveront accrus. (François Cornut-Gentille, séance du 26 mai 1998, Assemblée nationale)

En d'autres termes, c'est le monopole représentatif des élus qu'il s'agit ici de défendre. Enfin, ce sont les électeurs qui restent les maitres du jeu. En élisant des députés cumulants, ils votent en quelque sorte pour le cumul. La légitimité électorale est supérieure à celle de l'opinion.

La loi conduit à réduire les espaces de liberté auxquels aspirent les Français. C'est à eux de choisir : "Il existe, dans le domaine qui nous préoccupe, un maitre que nous respectons tous : le suffrage universel, un maitre qui n'hésite pas, lorsqu'il l'estime souhaitable, à faire connaitre sa volonté » Uacques Larché, séance du 27 octobre 1998, Sénat). Alain Vasselle va plus loin (séance du 28 octobre 1998, Sénat) : "Ce texte est une atteinte à la démocratie.»

\section{Proximité et intérêts corporatistes des professionnels de la politique}

La question de la proximité constitue donc une des pierres angulaires du débat. La proximité apparait comme un référent particulièrement plastique faisant l'objet d'investissements discursifs pluriels et justifiant des causes multiples, en l'occurrence ici opposées. L'analyse des débats permet aussi de

23. Un député, D. Paillé, demande pour maintenir le lien la constitutionnalisation du mode de scrutin (séance du 26 mai 1998). 
montrer comment l'exigence du terrain est intériorisée par les acteurs politiques et comment elle structure leur représentation de ce que doit être un bon élu. L'importance du terrain n'est contestée par aucun intervenant. Par-delà les oppositions manifestées, le débat révèle une croyance très largement partagée, à droite comme à gauche, celle de la nécessité du terrain et de la proximité, et l'idée selon laquelle cette légitimité est attachée à l'exercice de responsabilités locales. L'invocation de la proximité par la droite a d'autant plus de poids qu'elle est partagée et perçue comme une ressource de premier plan par la majorité des députés de gauche, où les maires sont majoritaires ${ }^{24}$. Si une partie des députés a défendu avec une telle âpreté la proximité, c'est qu'elle fait partie de l'idéologie professionnelle, qu'elle justifie leur utilité sociale et la dignité de leur fonction. L'argument de la proximité mobilisé par les partisans du cumul sert de justification normative à des situations acquises et à une concentration gratifiante des responsabilités entre quelques mains. Il tend à occulter les intérêts propres des professionnels de la politique et leur permet de «transformer des intérêts corporatistes déniés en intérêt général requis ${ }^{25}$. Tout se passe comme si était en jeu, dans le débat, la légitimation même du métier et qu'un accord se dégageait sur la nécessité pour les élus pris dans leur ensemble de ne pas se priver des ressources à la fois électorales et symboliques de la proximité. En défendant la proximité au nom de l'opinion, les élus défendent leurs intérêts propres.

\section{Ressources de la proximité et inamovibilité des élus}

L'idéologie du terrain apparait aussi prégnante à gauche qu'à droite ${ }^{26}$. Très rapidement, au fil du débat, la nécessité de ne pas couper l'élu national de ses bases locales s'est imposée. Le débat porte dès lors sur les modalités du maintien de ce lien. L'enjeu du projet s'est ainsi peu à peu déplacé : l'objectif est d'aboutir à une limitation du cumul des mandats qui ne coupe pas les élus nationaux des citoyens. Tout se passe comme s'il était inconcevable de porter atteinte à la proximité parce qu'elle touchait un aspect essentiel du métier

24. En octobre 1997, $56 \%$ des députés étaient maires, $39 \%$ conseillers généraux. 47 députés n'exerçaient que ce mandat (sources : Assemblée nationale, Trombinoscope). Robert Hue a milité lors du débat pour l'introduction d'un seuil de 100000 habitants au-delà duquel le cumul parlementaire-maire ne serait pas autorisé. Notons que le PC comptait alors 28 députésmaires et que 27 d'entre eux étaient maires d'une commune de moins de 100000 habitants.

25. P. Lehingue, 1999, art. cité, p. 101.

26. J.-M. Ayrault, président du groupe socialiste, avait en partie expliqué le nombre insuffisant de députés socialistes lors du vote de la loi sur le PACS par les difficultés à concilier la nécessaire proximité et le travail parlementaire. Il écrit ainsi : «Rien ne fera que le député puisse passer toute sa semaine à l'Assemblée. En effet, le député ne peut exercer les fonctions qui lui sont dévolues par la Constitution que s'il garde un contact étroit avec les citoyens. Pour cela, il doit les rencontrer. C'est ainsi qu'il peut ne pas être entre les mains de la technocratie et, en même temps, sentir battre le pouls du pays, donc, bien légiférer» (Libération, 4 novembre 1998). 
politique. Alors que cette disposition n'était initialement pas prévue, le gouvernement a imposé ${ }^{27}$ d'accorder une concession aux défenseurs du lien avec le terrain : si le lien maire-député est coupé, le député pourra rester adjoint, conseiller municipal, membre d'une assemblée départementale ou régionale et les mandats exercés au sein des structures intercommunales ne seront pas concernés par le dispositif de limitation du cumul. Ces fonctions doivent permettre «de maintenir un lien avec les réalités locales pour ceux qui ont des responsabilités nationales». Devant la vigueur des critiques, le rapporteur Bernard Roman rappelle, pour calmer les esprits, que le projet prévoit «le maintien de l'ancrage local» (séance du 26 mai 1998, Assemblée nationale). Ainsi, pour le ministre de l'Intérieur, Jean-Pierre Chevènement, «les parlementaires pourront continuer de participer activement aux assemblées locales, départementales ou régionales, y exercer des responsabilités et demeurer, s'ils le souhaitent, au contact des citoyens » (audition du ministre de l'Intérieur, commission des lois) ${ }^{28}$.

La conception du terrain que les parlementaires de gauche défendent est essentiellement institutionnelle, c'est-à-dire attachée à l'exercice de mandats locaux. Rares sont les parlementaires qui mettent en avant d'autres manières de faire la politique et ces derniers n'exercent pas de mandats locaux ou sont de jeunes députés. Guy Allouche, sénateur (séance du 19 octobre 1999), est isolé :

Il serait inexact, et surtout prétentieux, d'affirmer que la connaissance du terrain passe par l'exercice d'une fonction exécutive. La participation efficace aux travaux d'une assemblée, une bonne connaissance des problèmes n'impliquent aucunement la charge de l'exécutif. Si seul l'exécutif est au «cœur des réalités du terrain », à quoi servent alors les adjoints et les conseillers municipaux, les vice-présidents et les membres des commissions permanentes?

Pour la sénatrice socialiste Dinah Derycke (séance du 28 octobre 1998, Sénat) :

Le projet de loi n'interdit pas l'ancrage local des parlementaires [...] mais de toute façon, il nous faudra modifier notre conception de l'ancrage local et ne pas la limiter aux seuls mandats électoraux. L'ancrage d'un parlementaire peut également se faire par une participation à la vie quotidienne de nos concitoyens. L'ancrage d'un parlementaire peut très bien être assuré par la présidence d'associations soit locales soit nationales qui travaillent sur le terrain.

27. L'Express, 4 décembre 1997.

28. Rares sont les députés socialistes favorables à une coupure totale entre fonctions nationales et fonctions locales. On compte parmi eux Jean-Pierre Balligand, député-maire de Vervins, auteur d'un rapport pour le PS en mars 1997 sur la décentralisation, qui soutient l'idée de "structurer deux filières électives spécialisées : l'une à vocation nationale et l'autre locale, afin de respecter le caractère de chaque type de mandat et d'isoler la fonction législative des préoccupations locales, souvent trop imprégnées de particularisme» (J.-P. Balligand, 1998, Le Courrier des maires, 9 janvier). 
Si cette idéologie du terrain est largement partagée, c'est qu'elle engage la croyance que la proximité met en jeu des ressources essentielles pour la réélection et la recherche de l'inamovibilité électorale. Les orateurs de droite jouent de cette croyance. Plusieurs d'entre eux invitent les députés de gauche à ne pas se priver des ressources de la proximité qui met en jeu leur réélection, appelant leurs «collègues ${ }^{29}$ à ne pas mettre en cause les règles du jeu politique qui profitent à chacun des joueurs. Jean-Paul Delevoye (séance du 27 octobre 1998, Sénat) évoque sur un mode détourné les contraintes concurrentielles de la compétition électorale :

À suivre votre proposition, le risque est grand de voir le parlementaire, dorénavant exclu de l'exercice d'un exécutif local dans une circonscription de 100000 habitants, par exemple, percevoir immédiatement le maire d'une commune de 60000 habitants comme son futur concurrent. Le risque est grand de le voir s'empresser - pardonnez-moi l'expression - de le marquer à la culotte, provoquant alors la réaction du maire, qui marginaliserait le parlementaire.

Pascal Clément est encore plus explicite, appelant les députés de gauche à garder à l'esprit leurs intérêts bien compris :

En réalité, les Français apprécient un député en fonction de ses réalisations locales, de ce qu'il fait et propose localement [...]. Si vous attendez des Français qu'ils vous jugent sur votre travail à Paris, mes chers collègues, j’ai une bonne nouvelle pour vous : vous aurez tout le temps d'être élu local après, car vous ne siégerez plus ici la prochaine fois. (Séance du 26 mai 1998, Assemblée nationale)

C'est reconnaitre par là que ce travail de proximité consiste avant tout dans une activité de démarchage électoral et que le cumul joue un rôle essentiel dans la monopolisation des fonctions électives en constituant «un instrument de réduction de l'incertitude électorale $»^{30}$. Les polémiques, peu fréquentes on l'a dit, sur la façon de faire la politique, contraignent les acteurs en concurrence à lever le voile sur certaines pratiques occultées le plus souvent, à dévoiler les coulisses du jeu politique. Ce type de débat est propice à la levée des censures. L'accord, tacite en phase routinière, sur certaines règles du jeu officieuses, s'explicite provisoirement. Les règles du jeu, jusque-là occultées, sont portées au jour ${ }^{31}$. Dans cette séquence critique, l'univers de ce qui peut être dit semble se dilater sous l'effet de l'enjeu. Les parlementaires de droite appellent ainsi leurs collègues à une solidarité corporative qui doit maintenir les règles du

29. La récurrence, dans les débats, des formules oratoires comme «vous savez bien, chers collègues", mettant en jeu un implicite partagé par tous les hommes politiques un peu avisés et témoignant d'évidences partagées, est tout à fait éclairante de l'importance de l'enjeu.

30. G. Marrel, 2003, L'élu et son double, ouvr. cité, p. 49.

31. C'est un des intérêts des jeux d'Assemblée, même s'ils sont peu étudiés en science politique. Voir O. Nay, «Le travail politique à l'Assemblée. Note sur un champ de recherche trop longtemps déserté », Sociologie du travail, à paraitre. 
champ, les invite au respect d'une complicité objective qui, par-delà les luttes qui les opposent, fait tenir le champ et régit la concurrence qui s'y développe.

Ils le font d'autant plus qu'une large majorité des parlementaires de gauche exprime de grandes réserves à l'égard du projet ${ }^{32}$ et attend que le Sénat s'y oppose ${ }^{33}$. De nombreux orateurs font état de cette réticence et de ce calcul politique. Jacques Peyrat, sénateur RPR (séance du 27 octobre 1998) révèle les coulisses du débat :

Monsieur le président, monsieur le ministre, mes chers collègues, ayant eu le privilège de participer en tant que député à l'examen en première lecture des deux textes sur la limitation de l'exercice de plusieurs mandats, permettez-moi de vous faire part de ce que j'ai pu ressentir à l'époque, à savoir un profond embarras de tous les acteurs. Embarras d'un ministre, tout d'abord ancien député-maire de Belfort, qui était obligé de défendre un texte auquel il m'a semblé ne pas trop croire du fait, justement, de son expérience. Embarras d'une majorité, ensuite, majorité on ne peut plus plurielle qui allait des radicaux et des communistes, très attachés à la fonction de député-maire, au jeune député apparatchik socialiste tout heureux d'avoir trouvé un coupable : le cumulard, qu'il devait ainsi présenter à l'Être suprême : l'opinion. Embarras d'une droite enfin, il faut le dire, hésitant à s'opposer à une réforme qu'elle jugeait mauvaise mais que les médias présentaient comme populaire.

\section{Proximité et ressources symboliques du cumul}

Le débat permet également de mettre au jour le double horizon qui structure les pratiques politiques des élus cumulants. À entendre les députés parler de leur métier, l'opposition entre le local et le national, celles connexes entre le haut et le bas, le sérail et la société, le terrain et l'abstraction des discours politiques généraux, apparaissent comme un principe de classement particulièrement prégnant de leur entendement politique. Un accord se dessine pour ne pas mettre en cause ce principe de vision et de division qui structure leur rapport à la politique et organise leurs stratégies et leurs anticipations. C'est que le jeu politique qu'institue ce schème de perception permet aux élus de jouer sur deux registres territoriaux. On mesure ici à quel point le local gagne à être analysé comme une catégorie indigène des professionnels

32. La presse se fait l'écho de discussions virulentes au sein du groupe parlementaire socialiste. Les caciques du PS sont très réservés à l'égard du projet. Un jeune député socialiste évoque, à propos de ce qui fonde ces réticences, des «argumentaires de hobereau de province d'antan » (Libération, 17 décembre 1999).

33. Au terme du débat, le Sénat parvient à ne pas supprimer le cumul d'un mandat de député et d'un exécutif local. Le nouveau dispositif interdit le cumul des mandats de parlementaire et de député européen, réduit les possibilités de cumuler un mandat parlementaire avec un mandat ou une fonction exécutive locale. Le cumul des mandats est désormais limité à deux mandats électifs et le cumul des fonctions exécutives de maire et de président de conseil régional ou général est interdit. 
de la politique ${ }^{34}$. Si les élus contribuent à l'objectivation d'une dimension locale du politique et entretiennent la croyance dans son existence, c'est en partie parce qu'elle leur permet de mobiliser des ressources spécifiques. Pour se prévaloir du terrain et exciper de la proximité, il faut objectiver la distance. L'opposition entre local et national, qui est largement le fait des élus, prend ici tout son sens. Tout se passe comme si les élus locaux puisaient dans le local et les représentations qui lui sont attachées des ressources de légitimation qui leur font de plus en plus défaut sur le plan national. Les élus cherchent d'autant plus à être proches qu'ils tendent à s'appuyer, avec la professionnalisation politique, sur des ressources de plus en plus distinctives (la compétence, notamment). Autrement dit, il s'agit, à partir du local, de neutraliser les effets de clôture du champ politique, de restaurer la validité même du jeu politique et la division du travail politique qui en est au principe. Il revient ainsi aux élus de ne pas se priver du gisement de légitimité que recèle le local. La proximité, dans cette dimension ascendante, s'apparente à un discours d'autolégitimation. C'est dire si la défense du cumul des mandats, auquel les élus sont farouchement attachés, constitue un enjeu politique essentiel. II ne s'agit pas simplement de défendre une forme de concentration des pouvoirs dont la rentabilité électorale est forte, mais bien de conserver les profits symboliques attachés à la multipositionnalité. La défense du local et de la proximité transcende ainsi largement les clivages partisans. Les débats sur le cumul des mandats révèlent les intérêts communs qui lient les élus locaux des divers partis politiques 35 . Dans la mesure où c'est la légitimité générale du jeu politique qui est en jeu, les solidarités corporatives (l'appartenance au groupe des élus locaux et des professionnels de la politique de manière plus large) tendent à supplanter les affiliations partisanes.

34. J. Lagroye, 1993, «De l'objet local à l'horizon local des pratiques», dans A. Mabileau (dir.), À la recherche du local, Paris, L'Harmattan, p. 166-182.

35. Comme le note P. Le Lidec, «si le souci du consentement des élus aux réformes institutionnelles est aussi marqué, c'est qu'à travers elles se joue la légitimité des règles du jeu de la compétition politique. Chacun sait que de telles réformes touchent au cœur des intérêts propres des représentants» (P. Le Lidec, 2001, Les maires dans la République. L'Association des maires de France, élément constitutif des régimes politiques français depuis 1907, thèse de science politique, Université Paris 1). 